\title{
A GENERALIZATION OF STRAUBE'S THEOREM: EXISTENCE OF ABSOLUTELY CONTINUOUS INVARIANT MEASURES FOR RANDOM MAPS
}

MD. SHAFIQUL ISLAM, PAWEL GÓRA, AND ABRAHAM BOYARSKY

Received 29 February 2004 and in revised form 1 September 2004

A random map is a discrete-time dynamical system in which one of a number of transformations is randomly selected and applied at each iteration of the process. In this paper, we study random maps. The main result provides a necessary and sufficient condition for the existence of absolutely continuous invariant measure for a random map with constant probabilities and position-dependent probabilities.

\section{Introduction}

Random dynamical systems provide a useful framework for modeling and analyzing various physical, social, and economic phenomena. A random dynamical system of special interest is a random map where the process switches from one map to another according to fixed probabilities [5] or, more generally, position-dependent probabilities $[1,3,4]$. The existence and properties of invariant measures for random maps reflect their longterm behavior and play an important role in understanding their chaotic nature.

It is well known that if a map $\tau: I \rightarrow I, I=[0,1]$, is piecewise expanding, then it possesses an absolutely continuous invariant measure (ACIM) [2]. This result can be generalized to random maps where the condition of piecewise expanding is replaced by an average expanding condition where the weighting coefficients are the probabilities of switching $[3,4,5]$. Such results have been generalized in [1]. There are a number of interesting examples which do not fall into the average expanding condition for which the conditions of this paper may present a possible approach.

Consider the following simple random maps on $I$ :

$$
\tau_{1}(x)=\frac{x}{2}, \quad \tau_{2}(x)=\frac{(x+1)}{2},
$$

with constant probabilities $p_{1}$ and $p_{2} . \tau_{1}$ has an attracting fixed point at 0 , while $\tau_{2}$ has an attracting fixed point at 1 . Thus, neither $\tau_{1}$ nor $\tau_{2}$ has an ACIM, yet any random map based on these two maps has Lebesgue measure as its unique ACIM. This shows that a random map does not necessarily inherit the properties of the underlying maps. Consider now an expanding map $\tau_{1}$ on $I$ and the logistic map $\tau_{2}$ on $I$. Both maps have 
an ACIM, but the "average expanding" sufficiency condition for existence of an ACIM for the random map based on $\tau_{1}$ and $\tau_{2}$ fails since $\tau_{2}$ has regions of arbitrarily small slope. Hence, in general, we cannot conclude that even such a simple random map admits an ACIM.

The foregoing suggests the need for results that can establish existence of an ACIM directly for random maps. To this end we generalize a theorem of Straube [7], which provides a necessary and sufficient condition for existence of an ACIM of a nonsingular map, to random maps. We consider both random maps with constant probabilities and random maps with position-dependent probabilities.

In Section 2, we present the notation and summarize the results that we will need in the sequel. In Section 3, we prove the main result.

\section{Preliminaries}

Let $(X, \mathscr{B}, \lambda)$ be a measure space, where $\lambda$ is an underlying measure and let $\tau_{k}: X \rightarrow X, k=$ $1,2, \ldots, K$ be nonsingular transformations. A random map $T$ with constant probabilities is defined as

$$
T=\left\{\tau_{1}, \tau_{2}, \ldots, \tau_{K} ; p_{1}, p_{2}, \ldots, p_{K}\right\}
$$

where $\left\{p_{1}, p_{2}, \ldots, p_{K}\right\}$ is a set of constant probabilities. For any $x \in X, T(x)=\tau_{k}(x)$ with probability $p_{k}$ and for any nonnegative integer $N, T^{N}(x)=\tau_{k_{N}} \circ \tau_{k_{N-1}} \circ \cdots \circ \tau_{k_{1}}(x)$ with probability $\Pi_{j=1}^{N} p_{k_{j}}$. A $T$-invariant measure satisfies the following condition [6]:

$$
\mu(E)=\sum_{k=1}^{K} p_{k} \mu\left(\tau_{k}^{-1}(E)\right),
$$

for any $E \in \mathscr{B}$.

A position-dependent random map $T$ is defined as

$$
T=\left\{\tau_{1}, \tau_{2}, \ldots, \tau_{K} ; p_{1}(x), p_{2}(x), \ldots, p_{K}(x)\right\}
$$

where $\left\{p_{1}(x), p_{2}(x), \ldots, p_{K}(x)\right\}$ is a set of position-dependent probabilities, that is, $\sum_{k=1}^{K} p_{k}(x)=1$, for any $x \in X, T(x)=\tau_{k}(x)$ with probability $p_{k}(x)$ and for any nonnegative integer $N, T^{N}(x)=\tau_{k_{N}} \circ \tau_{k_{N-1}} \circ \cdots \circ \tau_{k_{1}}(x)$ with probability

$$
p_{k_{N}}\left(\tau_{k_{N-1}} \circ \cdots \circ \tau_{k_{1}}(x)\right) p_{k_{N-1}}\left(\tau_{k_{N-2}} \circ \cdots \circ \tau_{k_{1}}(x)\right) \cdots p_{k_{1}}(x) .
$$

In [2], it was proved that a $T$-invariant measure $\mu$ is given by

$$
\mu(E)=\sum_{k=1}^{K} \int_{\tau_{k}^{-1}(E)} p_{k}(x) d \mu(x),
$$

for any measurable set $E \in \mathscr{B}$.

We now recall some definitions and results from $[6,7]$ which will be used to prove our main results in Section 3. 
Definition 2.1. A set function $\phi: \mathscr{B} \rightarrow R$ is finitely additive measure if

(i) $-\infty<\phi(E)<\infty$, for all $E \in \mathscr{B}$;

(ii) $\phi(\varnothing)=0$;

(iii) $\sup _{E \in \mathscr{P}}|\phi(E)|<\infty$;

(iv) $\phi\left(E_{1} \cup E_{2}\right)=\phi\left(E_{1}\right)+\phi\left(E_{2}\right)$, for all $E_{1}, E_{2} \in \mathscr{B}$ such that $E_{1} \cap E_{2}=\varnothing$.

Definition 2.2. A finitely additive positive measure $\mu$ is purely additive measure if every countably additive measure $\nu \geq 0, \nu \leq \mu$ is identically zero.

Theorem 2.3 [7]. Let $\phi$ be finitely additive (positive) measure. Then $\phi$ has a unique representation $\phi=\phi_{c}+\phi_{p}$, where $\phi_{c}$ is countably additive $\left(\phi_{c} \geq 0\right)$ and $\phi_{p}$ is purely additive $\left(\phi_{p} \geq 0\right)$.

Lемма 2.4 [7]. If $\mu$ is a finitely additive positive measure on $\mathscr{B}$, then $\mu_{c}$ is the greatest measure among countably additive measures $\nu$ with $0 \leq \nu \leq \mu$.

THeOREM 2.5 [7]. Let $\phi$ be a finitely additive positive measure on a $\sigma$-algebra $\mathscr{B}$ and let $\nu$ be a countably additive positive measure on $\mathscr{B}$. Then, there exists a decreasing sequence $\left\{E_{n}\right\}_{n \geq 1}$ of elements of $\mathscr{B}$ such that $\lim _{n \rightarrow \infty} \nu\left(E_{n}\right)=0$ and $\phi\left(E_{n}\right)=\phi(X)$.

Theorem $2.6[6]$. Let $(X, B, \lambda)$ be a measure space with normalized measure $\lambda$, and let $f: X \rightarrow X$ be a nonsingular transformation. Then, the following conditions are equivalent:

(i) there exists an $f$-invariant normalized measure $\mu$ which is absolutely continuous with respect to $\lambda$;

(ii) there exists $\delta>0$, and $\alpha, 0<\alpha<1$ such that

$$
\lambda(E)<\delta \Longrightarrow \sup _{k \in N} \lambda\left(f^{-k}(E)\right)<\alpha, \quad E \in \mathscr{B} .
$$

\section{Existence of absolutely continuous invariant measures}

In this section, we prove necessary and sufficient conditions for existence of an absolutely continuous invariant measure for random maps. For notational convenience, we consider $K=2$, that is, we consider only two transformations $\tau_{1}, \tau_{2}$. The proofs for larger number of maps are analogous. We first consider random maps with constant probabilities, then random maps with position-dependent probabilities.

Theorem 3.1. Let $(X, \mathscr{B}, \lambda)$ be a measure space with normalized measure $\lambda$ and let $\tau_{i}: X \rightarrow$ $X, i=1,2$ be nonsingular transformations. Consider the random map $T=\left\{\tau_{1}, \tau_{2} ; p_{1}, p_{2}\right\}$ with constant probabilities $p_{1}, p_{2}$. Then, there exists a normalized absolutely continuous (w.r.t. $\lambda$ ) T-invariant measure $\mu$ if and only if there exists $\delta>0$ and $0<\alpha<1$ such that for any measurable set $E$ and any positive integer $k, \lambda(E)<\delta$ implies

$$
\begin{gathered}
p_{1} \lambda\left(\tau_{1}^{-1}(E)\right)+p_{2} \lambda\left(\tau_{2}^{-1}(E)\right)<\alpha ; \\
p_{1}^{2} \lambda\left(\tau_{1}^{-2}(E)\right)+p_{1} p_{2} \lambda\left(\tau_{2}^{-1} \tau_{1}^{-1}(E)\right)+p_{1} p_{2} \lambda\left(\tau_{1}^{-1} \tau_{2}^{-1}(E)\right)+p_{2}^{2} \lambda\left(\tau_{2}^{-2}(E)\right)<\alpha ; \\
\vdots \\
\sum_{\left(i_{1}, i_{2}, i_{3}, \ldots, i_{k}\right)} p_{i_{1}} p_{i_{2}} \cdots p_{i_{k}} \lambda\left(\tau_{i_{1}}^{-1} \tau_{i_{2}}^{-1} \cdots \tau_{i_{k}}^{-1}(E)\right)<\alpha .
\end{gathered}
$$


To prove this theorem, we first prove the following two lemmas.

Lemma 3.2. Let $(X, \mathscr{B}, \lambda)$ be a probability measure space and let $\mu$ be absolutely continuous with respect to $\lambda, \mu=f \cdot \lambda$, for $f$ an $L^{1}(X, \mathscr{B}, \lambda)$ function. Then, there exists a constant $M \geq 0$ and a measurable set $A_{0}$ such that $\mu\left(A_{0}\right) \leq 1 / 10$ and $f \leq M$ on $X \backslash A_{0}$.

Proof. Consider the following sets:

$$
B_{n}=\{x \in X: n \leq f(x)<n+1\}, \quad n=0,1, \ldots
$$

Clearly, $\left\{B_{n}\right\}$ are disjoint measurable sets and $X=\cup_{n=0}^{\infty} B_{n}$ and $1=\mu(X)=\sum_{n=0}^{\infty} \mu\left(B_{n}\right)$. Thus, there exists an $M \geq 0$ such that $\sum_{n=M}^{\infty} \mu\left(B_{n}\right)<1 / 10$. Let $A_{0}=\bigcup_{n=M}^{\infty} B_{n}$. Then on $X \backslash \bigcup_{n=M}^{\infty} B_{n}, f(x) \leq M$.

For any measure $\phi$, any integer $k$, and any measurable set $E$, define

$$
\Lambda_{k}^{\phi}(E):=\sum_{\left(i_{1}, i_{2}, i_{3}, \ldots, i_{k}\right)} p_{i_{1}} p_{i_{2}} \cdots p_{i_{k}} \phi\left(\tau_{i_{1}}^{-1} \tau_{i_{2}}^{-1} \cdots \tau_{i_{k}}^{-1}(E)\right) .
$$

It can be easily shown that $\Lambda_{k}^{\lambda}$ and $\Lambda_{k}^{\mu}$ are normalized measures and $\Lambda_{k}^{\mu}$ are measures absolutely continuous with respect to $\Lambda_{k}^{\lambda}$.

Lemma 3.3. Let $M$ be the constant from the previous lemma and let $\delta$ be such that $M \delta+$ $1 / 10<1 / 4$. Then, for any $n \geq 1$, and any measurable set $A, \Lambda_{n}^{\lambda}(A)<\delta \Rightarrow \Lambda_{n}^{\mu}(A)<1 / 4$.

Proof. Let $M$ and $A_{0}$ be as in the previous lemma. We have

$$
\begin{aligned}
\Lambda_{n}^{\mu}(A)= & \sum_{\left(i_{1}, i_{2}, i_{3}, \ldots, i_{n}\right)} p_{i_{1}} p_{i_{2}} \cdots p_{i_{n}} \mu\left(\tau_{i_{1}}^{-1} \tau_{i_{2}}^{-1} \cdots \tau_{i_{n}}^{-1}(A)\right) \\
= & \sum_{\left(i_{1}, i_{2}, i_{3}, \ldots, i_{n}\right)} p_{i_{1}} p_{i_{2}} \cdots p_{i_{n}} \mu\left(\tau_{i_{1}}^{-1} \tau_{i_{2}}^{-1} \cdots \tau_{i_{n}}^{-1}(A) \cap A_{0}\right) \\
& +\sum_{\left(i_{1}, i_{2}, i_{3}, \ldots, i_{n}\right)} p_{i_{1}} p_{i_{2}} \cdots p_{i_{n}} \mu\left(\tau_{i_{1}}^{-1} \tau_{i_{2}}^{-1} \cdots \tau_{i_{n}}^{-1}(A) \cap\left(X \backslash A_{0}\right)\right) \\
\leq & \sum_{\left(i_{1}, i_{2}, i_{3}, \ldots, i_{n}\right)} p_{i_{1}} p_{i_{2}} \cdots p_{i_{n}} \frac{1}{10}+\sum_{\left(i_{1}, i_{2}, i_{3}, \ldots, i_{n}\right)} p_{i_{1}} p_{i_{2}} \cdots p_{i_{n}} M \lambda\left(\tau_{i_{1}}^{-1} \tau_{i_{2}}^{-1} \cdots \tau_{i_{n}}^{-1}(A)\right) \\
\leq & \frac{1}{10}+M \Lambda_{n}^{\lambda}(A)<\frac{1}{10}+M \delta<\frac{1}{4} .
\end{aligned}
$$

Proof of Theorem 3.1. Suppose

$$
\mu(E)=\sum_{i=1}^{2} p_{i} \mu\left(\tau_{i}^{-1}(E)\right), \quad E \in B, \quad \mu(X)=1, \quad \mu \ll \lambda .
$$

We want to prove that there exist $\delta>0,0<\alpha<1$ such that for any $E \in \mathscr{B}$ and for any positive integer $k$,

$$
\lambda(E)<\delta \Longrightarrow \Lambda_{k}^{\lambda}(E)<\alpha
$$


Suppose not. Then, for any $\alpha, 0<\alpha<1$, there exists $E \in \mathscr{B}$ and there exists a positive integer $n_{0}$ such that

$$
\lambda(E)<\delta \Longrightarrow \Lambda_{n_{0}}^{\lambda}(E)>\alpha
$$

where $E \in \mathscr{R}$.

Choose $\delta>0$ such that $M \delta+1 / 10<1 / 4$, where $M$ is the constant of Lemma 3.2. Let $n_{0}$ be the index corresponding to $\delta$ in formula (3.6). Then by Lemma 3.2, we have, for $A \in \mathscr{B}$,

$$
\begin{gathered}
\lambda(A)<\delta \Longrightarrow \mu(A)<\frac{1}{4} ; \\
\Lambda_{n_{0}}^{\lambda}(A)<\delta \Longrightarrow \Lambda_{n_{0}}^{\mu}(A)<\frac{1}{4} .
\end{gathered}
$$

Let $\alpha=1-\delta / 2$. Then,

$$
\Lambda_{n_{0}}^{\lambda}(X \backslash E)=1-\Lambda_{n_{0}}^{\lambda}(E)<1-1+\delta=\delta .
$$

From our choice of $\delta$, we get

$$
\Lambda_{n_{0}}^{\mu}(X \backslash E)<\frac{1}{4}
$$

Since $\mu$ is invariant, we have

$$
\mu(X \backslash E)=\Lambda_{n_{0}}^{\mu}(X \backslash E)<\frac{1}{4} .
$$

Thus,

$$
1=\mu(X)=\mu(E)+\mu(X \backslash E)<\frac{1}{4}+\frac{1}{4},
$$

a contradiction.

Conversely, suppose that there exists $\delta>0$ and $0<\alpha<1$ such that for any measurable set $E$ and any positive integer $k, \lambda(E)<\delta$ implies

$$
\begin{gathered}
p_{1} \lambda\left(\tau_{1}^{-1}(E)\right)+p_{2} \lambda\left(\tau_{2}^{-1}(E)\right)<\alpha \\
p_{1}^{2} \lambda\left(\tau_{1}^{-2}(E)\right)+p_{1} p_{2} \lambda\left(\tau_{2}^{-1} \tau_{1}^{-1}(E)\right)+p_{1} p_{2} \lambda\left(\tau_{1}^{-1} \tau_{2}^{-1}(E)\right)+p_{2}^{2} \lambda\left(\tau_{2}^{-2}(E)\right)<\alpha \\
\vdots \\
\sum_{\left(i_{1}, i_{2}, i_{3}, \ldots, i_{k}\right)} p_{i_{1}} p_{i_{2}} \cdots p_{i_{k}} \lambda\left(\tau_{i_{1}}^{-1} \tau_{i_{2}}^{-1} \cdots \tau_{i_{k}}^{-1}(E)\right)<\alpha .
\end{gathered}
$$

We want to show that there exists a measure $\mu$ such that $\mu(E)=\sum_{i=1}^{2} p_{i} \mu\left(\tau_{i}^{-1}(E)\right), E \in \mathscr{B}$, $\mu(X)=1$ and $\mu \ll \lambda$.

Consider the measures $\lambda_{n}$ defined by

$$
\lambda_{n}(E):=\frac{1}{n} \sum_{k=0}^{n-1} \Lambda_{k}^{\lambda}(E), \quad E \in \mathscr{B} .
$$


It can be shown that for all $n, \lambda_{n}$ are normalized measures. Moreover, if $\lambda(E)=0$, then

$$
\begin{aligned}
\lambda_{n}(E)= & \lambda(E)+p_{1} \lambda\left(\tau_{1}^{-1}(E)\right)+p_{2} \lambda\left(\tau_{2}^{-1}(E)\right) \\
& +p_{1}^{2} \lambda\left(\tau_{1}^{-2}(E)\right)+p_{1} p_{2} \lambda\left(\tau_{2}^{-1} \tau_{1}^{-1}(E)\right)+p_{1} p_{2} \lambda\left(\tau_{1}^{-1} \tau_{2}^{-1}(E)\right)+p_{2}^{2} \lambda\left(\tau_{2}^{-2}(E)\right) \\
& +\cdots+\sum_{\left(i_{1}, i_{2}, i_{3}, \ldots, i_{n}\right)} p_{i_{1}} p_{i_{2}} \cdots p_{i_{n}} \lambda\left(\tau_{i_{1}}^{-1} \tau_{i_{2}}^{-1} \cdots \tau_{i_{n}}^{-1}(E)\right)=0,
\end{aligned}
$$

by nonsingularity of $\tau_{1}$ and $\tau_{2}$. Hence $\lambda_{n} \ll \lambda$. We imbed $\lambda_{n}$ in the dual space $L_{\infty}(\lambda)^{*}$ of $L_{\infty}(\lambda)$ in the following way:

$$
g_{n}(f)=\int_{X} f d \lambda_{n}, \quad f \in L_{\infty}(\lambda)
$$

For every $n$,

$$
\left|g_{n}(f)\right|=\left|\int_{X} f d \lambda_{n}\right| \leq\|f\|_{\infty} \int_{X} d \lambda_{n}=\|f\|_{\infty}
$$

Hence, for each $n,\left\|g_{n}\right\| \leq 1$. Thus, the $\lambda_{n}$ can be thought of as elements of the unit ball of $L_{\infty}(\lambda)^{*}$. This unit ball is weak*-compact by Alaoglu's theorem [7]. Let $v$ be a cluster point in the weak*-topology of $L_{\infty}(\lambda)^{*}$ of the sequence $\left(\lambda_{n}\right)_{n \geq 1}$.

Define a set function $\mu$ on $\mathscr{B}$ by

$$
\mu(E)=\nu\left(\chi_{E}\right)
$$

We claim that $\mu$ is finitely additive, bounded and it vanishes on sets of $\lambda$-measure zero: $\mu(\varnothing)=\nu(\chi \varnothing)=\nu(0)=0$, since $\nu$ is a linear functional. For any $E \in \mathscr{B}$,

$$
\begin{aligned}
\mu(E) & =\nu\left(\chi_{E}\right)=\lim _{s \rightarrow \infty} g_{n_{s}}\left(\chi_{E}\right)=\lim _{s \rightarrow \infty} \int_{E} d \lambda_{n_{s}}=\lim _{s \rightarrow \infty} \lambda_{n_{s}}(E) \\
& =\lim _{s \rightarrow \infty} \frac{1}{n_{s}} \sum_{k=0}^{n_{s}-1} \Lambda_{k}^{\lambda}(E) \geq 0,
\end{aligned}
$$

since $\Lambda_{k}^{\lambda}$ is a measure. Thus,

$$
0 \leq \mu(E) \leq \mu(X)=\lim _{s \rightarrow \infty} \lambda_{n_{s}}(X)=1 .
$$

Now,

$$
\begin{aligned}
\mu\left(\bigcup_{i=1}^{m} E_{i}\right) & =\lim _{s \rightarrow \infty} \lambda_{n_{s}}\left(\bigcup_{i=1}^{m} E_{i}\right)=\lim _{s \rightarrow \infty} \sum_{i=1}^{m} \lambda_{n_{s}}\left(E_{i}\right) \\
& =\sum_{i=1}^{m} \lim _{s \rightarrow \infty} \lambda_{n_{s}}\left(E_{i}\right)=\sum_{i=1}^{m} \mu\left(E_{i}\right) .
\end{aligned}
$$

Let $\lambda(E)=0$. Then $\mu(E)=\lim _{s \rightarrow \infty} \lambda_{n_{s}}(E)=0$, because $\lambda_{n_{s}} \ll \lambda$. Hence, $\mu$ is finitely additive, bounded, and it vanishes on sets of $\lambda$-measure zero. 
$\mu$ is $T$-invariant:

$$
\begin{aligned}
\mu(E)= & \lim _{s \rightarrow \infty} \lambda_{n_{s}}(E)=\lim _{s \rightarrow \infty} \frac{1}{n_{s}} \sum_{k=0}^{n_{s}-1} \Lambda_{k}^{\lambda}(E) \\
= & \Lambda_{0}^{\lambda}(E)+\Lambda_{1}^{\lambda}(E)+\cdots+\Lambda_{n_{s}-1}^{\lambda}(E) \\
= & \lim _{s \rightarrow \infty} \frac{1}{n_{s}}\left[\lambda(E)+p_{1} \lambda\left(\tau_{1}^{-1}(E)\right)+p_{2} \lambda\left(\tau_{2}^{-1}(E)\right)\right. \\
& \left.+\cdots+\sum_{\left(i_{1}, i_{2}, i_{3}, \ldots, i_{n_{s}-1}\right)} p_{i_{1}} p_{i_{2}} \cdots p_{i_{n_{s}-1}} \lambda\left(\tau_{i_{1}}^{-1} \tau_{i_{2}}^{-1} \cdots \tau_{i_{n_{s}-1}}^{-1}(E)\right)\right] .
\end{aligned}
$$

On the other hand,

$$
\begin{aligned}
& \sum_{i=1}^{2} p_{i} \mu\left(\tau_{i}^{-1}(E)\right)=p_{1} \mu\left(\tau_{1}^{-1}(E)\right)+p_{2} \mu\left(\tau_{2}^{-1}(E)\right) \\
& =p_{1} \lim _{s \rightarrow \infty} \frac{1}{n_{s}} \sum_{k=0}^{n_{s}-1} \Lambda_{k}^{\lambda}\left(\tau_{1}^{-1}(E)\right)+p_{2} \lim _{s \rightarrow \infty} \frac{1}{n_{s}} \sum_{k=0}^{n_{s}-1} \Lambda_{k}^{\lambda}\left(\tau_{2}^{-1}(E)\right) \\
& =\lim _{s \rightarrow \infty} \frac{1}{n_{s}}\left[p _ { 1 } \left\{\lambda\left(\tau_{1}^{-1}(E)\right)+p_{1} \lambda\left(\tau_{1}^{-2}(E)\right)+p_{2} \lambda\left(\tau_{2}^{-1} \tau_{1}^{-1}(E)\right)+\cdots\right.\right. \\
& \left.+\sum_{\left(i_{1}, i_{2}, i_{3}, \ldots, i_{n_{s}-1}\right)} p_{i_{1}} p_{i_{2}} \cdots p_{i_{n_{s}-1}} \lambda\left(\tau_{i_{1}}^{-1} \tau_{i_{2}}^{-1} \cdots \tau_{i_{n_{s}-1}}^{-1}\left(\tau_{1}^{-1}(E)\right)\right)\right\} \\
& +p_{2}\left\{\lambda\left(\tau_{2}^{-1}(E)\right)+p_{1} \lambda\left(\tau_{1}^{-1} \tau_{2}^{-1}(E)\right)+p_{2} \lambda\left(\tau_{2}^{-2}(E)\right)+\cdots\right. \\
& \left.\left.+\sum_{\left(i_{1}, i_{2}, i_{3}, \ldots, i_{n_{s}-1}\right)} p_{i_{1}} p_{i_{2}} \cdots p_{i_{n_{s}-1}} \lambda\left(\tau_{i_{1}}^{-1} \tau_{i_{2}}^{-1} \cdots \tau_{i_{n_{s}-1}}^{-1}\left(\tau_{2}^{-1}(E)\right)\right)\right\}\right] \\
& =\lim _{s \rightarrow \infty} \frac{1}{n_{s}}\left[p_{1} \lambda\left(\tau_{1}^{-1}(E)\right)+p_{1}^{2} \lambda\left(\tau_{1}^{-2}(E)\right)+p_{1} p_{2} \lambda\left(\tau_{2}^{-1} \tau_{1}^{-1}(E)\right)+\cdots\right. \\
& +p_{1} \sum_{\left(i_{1}, i_{2}, i_{3}, \ldots, i_{n_{s}-1}\right)} p_{i_{1}} p_{i_{2}} \cdots p_{i_{n_{s}-1}} \lambda\left(\tau_{i_{1}}^{-1} \tau_{i_{2}}^{-1} \cdots \tau_{i_{n_{s}-1}}^{-1}\left(\tau_{1}^{-1}(E)\right)\right) \\
& +p_{2} \lambda\left(\tau_{2}^{-1}(E)\right)+p_{2} p_{1} \lambda\left(\tau_{1}^{-1} \tau_{2}^{-1}(E)\right)+p_{2}^{2} \lambda\left(\tau_{2}^{-2}(E)\right)+\cdots \\
& \left.+p_{2} \sum_{\left(i_{1}, i_{2}, i_{3}, \ldots, i_{n_{s}-1}\right)} p_{i_{1}} p_{i_{2}} \cdots p_{i_{n_{s}-1}} \lambda\left(\tau_{i_{1}}^{-1} \tau_{i_{2}}^{-1} \cdots \tau_{i_{n_{s}-1}}^{-1}\left(\tau_{2}^{-1}(E)\right)\right)\right] .
\end{aligned}
$$

Clearly,

$$
\mu(E)=\sum_{i=1}^{2} p_{i} \mu\left(\tau_{i}^{-1}(E)\right) .
$$


Thus, we have shown that $\mu$ is a finitely additive $T$-invariant measure. By Theorem $2.3, \mu$ has a unique representation

$$
\mu=\mu_{c}+\mu_{p}
$$

where $\mu_{c}$ is countably additive and $\mu_{c} \geq 0$ and $\mu_{p}$ is purely additive and $\mu_{p} \geq 0$. We claim that $\mu_{c} \neq 0$. Suppose $\mu_{c}=0$. Then by Theorem 2.5 there exists a decreasing sequence $\left\{E_{n}\right\}_{n \geq 1}$ of elements of $\mathscr{B}$ such that $\lim _{n \rightarrow \infty} \lambda\left(E_{n}\right)=0$ and $\mu\left(E_{n}\right)=\mu(X)=1$. Thus, there exists an integer $n_{0}$ such that for all $n \geq n_{0}, \lambda\left(E_{n}\right)<\delta$ and, as a consequence of our hypothesis, we have, for all $k$,

$$
\Lambda_{k}^{\lambda}\left(E_{n}\right)<\alpha
$$

Hence

$$
\lambda_{k}\left(E_{n}\right)<\alpha, \quad k=1,2,3, \ldots
$$

Thus, $\mu\left(E_{n}\right)=\lim _{s \rightarrow \infty} g_{n_{s}}\left(E_{n}\right)<\alpha<1$, a contradiction. Now,

$$
\begin{aligned}
\mu(E) & =p_{1} \mu\left(\tau_{1}^{-1}(E)\right)+p_{2} \mu\left(\tau_{2}^{-1}(E)\right) \\
& =p_{1}\left\{\mu_{c}\left(\tau_{1}^{-1}(E)\right)+\mu_{p}\left(\tau_{1}^{-1}(E)\right)\right\}+p_{2}\left\{\mu_{c}\left(\tau_{2}^{-1}(E)\right)+\mu_{p}\left(\tau_{2}^{-1}(E)\right)\right\} \\
& =\left\{p_{1} \mu_{c}\left(\tau_{1}^{-1}(E)\right)+p_{2} \mu_{c}\left(\tau_{2}^{-1}(E)\right)\right\}+\left\{p_{1} \mu_{p}\left(\tau_{1}^{-1}(E)\right)+p_{2} \mu_{p}\left(\tau_{2}^{-1}(E)\right)\right\} .
\end{aligned}
$$

Clearly $m: \mathscr{B} \rightarrow R$, defined by

$$
m(E)=p_{1} \mu_{c}\left(\tau_{1}^{-1}(E)\right)+p_{2} \mu_{c}\left(\tau_{2}^{-1}(E)\right)
$$

is a countably additive measure, and $m \leq \mu$. Thus, by Lemma 2.4, we have $m \leq \mu_{c}$ and hence

$$
E \mapsto \mu_{c}(E)-m(E)=\mu_{c}(E)-\left\{p_{1} \mu_{c}\left(\tau_{1}^{-1}(E)\right)+p_{2} \mu_{c}\left(\tau_{2}^{-1}(E)\right)\right\}
$$

is a positive measure. But this measure has total mass zero. Hence, it is a zero measure. Thus $\mu_{c}$ is $T$-invariant. Because $\mu$ vanishes on sets of $\lambda$-measure zero and $0 \leq \mu_{c} \leq \mu$, we have $\mu_{c} \ll \lambda$. Finally, $\gamma(E)=\mu_{c}(E) / \mu_{c}(X)$ is normalized, $T$-invariant, and absolutely continuous with respect to $\lambda$.

We now state the analogous result for position-dependent random maps.

Theorem 3.4. Let $(X, B, \lambda)$ be a measure space with normalized measure $\lambda$ and let $\tau_{i}: X \rightarrow$ $X, i=1,2$ be nonsingular transformations. Consider the random map $T=\left\{\tau_{1}, \tau_{2} ; p_{1}, p_{2}\right\}$ with position-dependent probabilities $p_{1}, p_{2}$. Then there exists a normalized absolutely continuous (w.r.t. $\lambda$ ) T-invariant measure $\mu$ if and only if there exists $\delta>0$ and $0<\alpha<1$ such 
that for any measurable set $E$ and any positive integer $k, \lambda(E)<\delta$ implies

$$
\begin{gathered}
\int_{\tau_{1}^{-1}(E)} p_{1}(x) d \lambda+\int_{\tau_{2}^{-1}(E)} p_{2}(x) d \lambda<\alpha \\
\int_{\tau_{1}^{-2}(E)} p_{1}(x) p_{1}\left(\tau_{1}(x)\right) d \lambda+\int_{\tau_{2}^{-1} \tau_{1}^{-1}(E)} p_{1}(x) p_{2}\left(\tau_{1}(x)\right) d \lambda \\
+\int_{\tau_{1}^{-1} \tau_{2}^{-1}(E)} p_{2}(x) p_{1}\left(\tau_{2}(x)\right) d \lambda+\int_{\tau_{2}^{-2}(E)} p_{2}(x) p_{2}\left(\tau_{2}(x)\right) d \lambda<\alpha \\
\vdots \\
\sum_{\left(i_{1}, i_{2}, i_{3}, \ldots, i_{k}\right)} \int_{\tau_{i_{1}}^{-1} \tau_{i_{2}}^{-1} \cdots \tau_{i_{k}}^{-1}(E)} p_{i_{1}}(x) p_{i_{2}}\left(\tau_{i_{1}}(x)\right) \cdots p_{i_{k}}\left(\tau_{i_{1}} \tau_{i_{2}} \cdots \tau_{i_{k-1}}(x)\right) d \lambda<\alpha .
\end{gathered}
$$

Proof. The proof is analogous to the proof of Theorem 3.1.

\section{Acknowledgments}

The research was supported by NSERC grant. The first author was supported by the Department of Mathematics, Concordia University.

\section{References}

[1] W. Bahsoun, P. Góra, and A. Boyarsky, Markov switching for position dependent random maps with application to forecasting in financial markets, SIAM journal on Applied Dynamical Systems 4 (2005), no. 2, 391-406.

[2] A. Boyarsky and P. Góra, Laws of Chaos, Probability and Its Applications, Birkhaüser Boston, Massachusetts, 1997.

[3] P. Góra and A. Boyarsky, Absolutely continuous invariant measures for random maps with position dependent probabilities, J. Math. Anal. Appl. 278 (2003), no. 1, 225-242.

[4] - Attainable densities for random maps, preprint.

[5] S. Pelikan, Invariant densities for random maps of the interval, Trans. Amer. Math. Soc. 281 (1984), no. 2, 813-825.

[6] E. Straube, On the existence of invariant, absolutely continuous measures, Comm. Math. Phys. 81 (1981), no. 1, 27-30.

[7] K. Yosida and E. Hewitt, Finitely additive measures, Trans. Amer. Math. Soc. 72 (1952), no. 11, $46-66$.

Md. Shafiqul Islam: Department of Mathematics and Statistics, Concordia University, 7141 Sherbrooke Street West, Montreal, Quebec, Canada H4B 1R6

E-mail address: shaf_is@alcor.concordia.ca

Pawel Góra: Department of Mathematics and Statistics, Concordia University, 7141 Sherbrooke Street West, Montreal, Quebec, Canada H4B 1R6

E-mail address: pgora@vax2.concordia.ca

Abraham Boyarsky: Department of Mathematics and Statistics, Concordia University, 7141 Sherbrooke Street West, Montreal, Quebec, Canada H4B 1R6

E-mail address: boyar@vax2.concordia.ca 


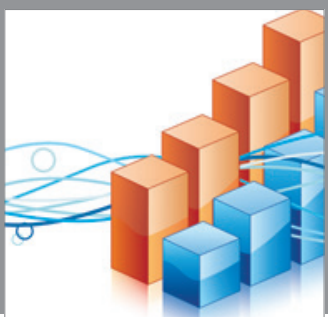

Advances in

Operations Research

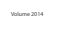

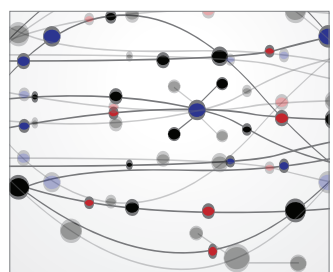

\section{The Scientific} World Journal
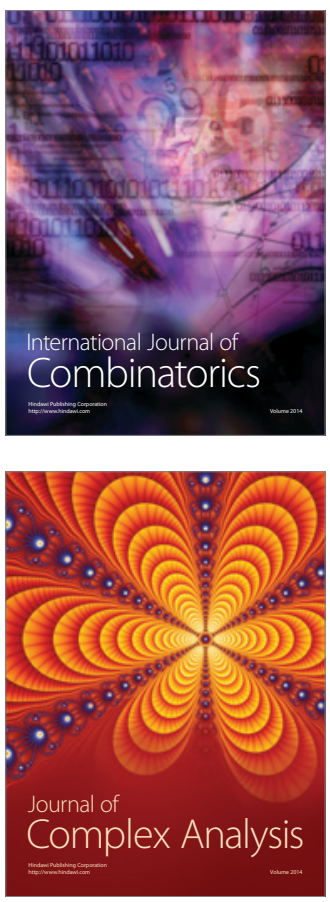

International Journal of

Mathematics and

Mathematical

Sciences
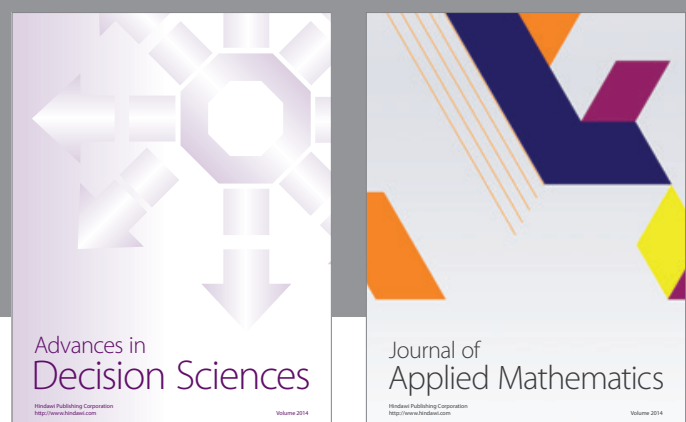

Journal of

Applied Mathematics
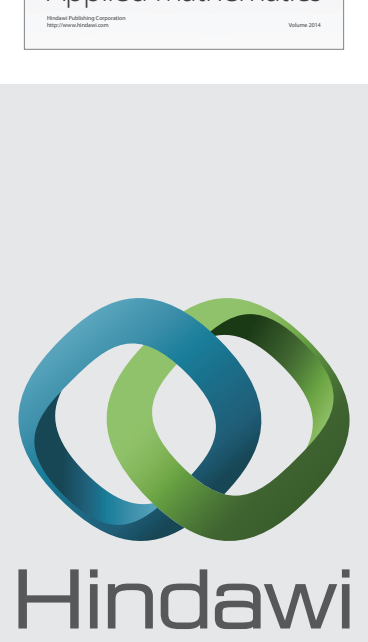

Submit your manuscripts at http://www.hindawi.com
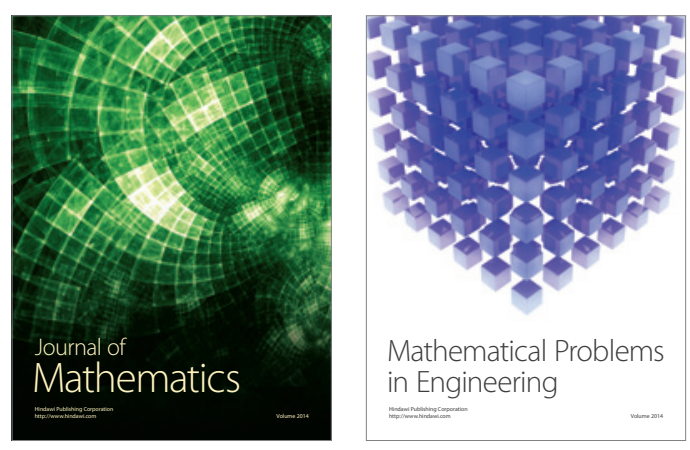

Mathematical Problems in Engineering
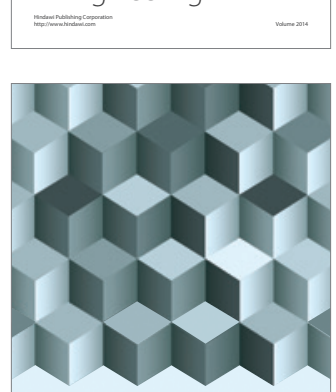

Journal of

Function Spaces
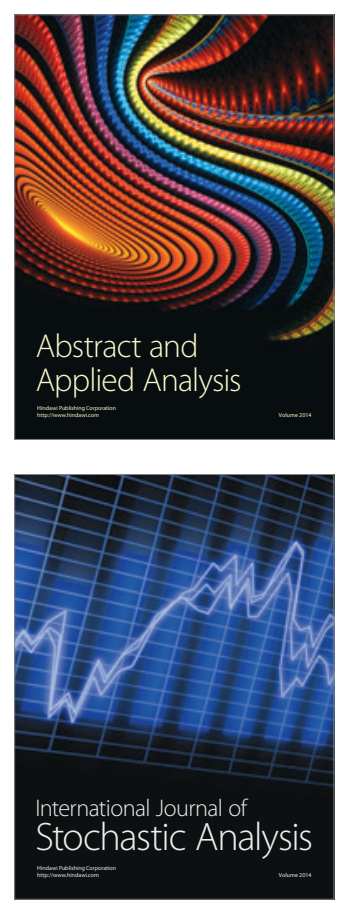

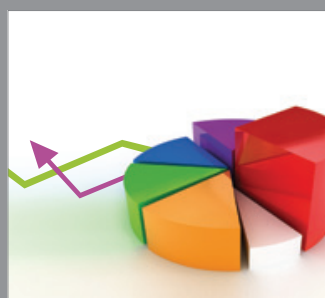

ournal of

Probability and Statistics

Promensencen
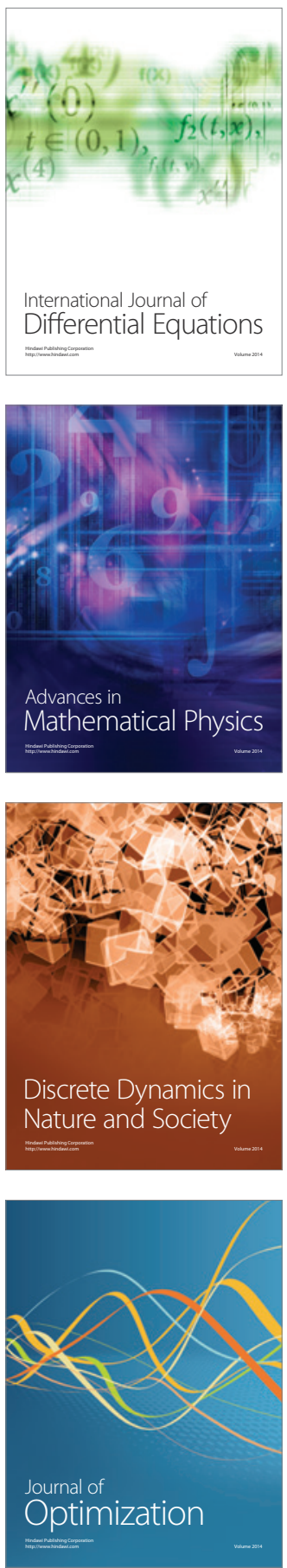